\title{
A New millimeter-wave micro-fluidic Temperature sensor for wireless passive radar interrogation
}

\author{
S. Bouaziz, F. Chebila, A. Traille, P. Pons, H. Aubert \\ ${ }^{1}$ CNRS, LAAS, 7 avenue du colonel Roche \\ Univ de Toulouse, UPS, INP, LAAS \\ F-31400, Toulouse, France \\ sbouaziz@laas fr
}

\begin{abstract}
This paper presents a micro-fluidic temperature sensor that uses a variable radar echo to measure temperature at Ka-frequency band. The device is made up of a planar gap capacitor with a micro-fluidic channel situated between the plates. As the temperature changes, the water level in the channel moves across the capacitor plates. This rising level of high permittivity liquid within the capacitor modifies the capacitance and as a result modifies the scattering parameter S11. The sensor can then be integrated with an antenna and interrogated at distance by a reader. The detected radar echo level changes in proportion to temperature when illuminated by the wave reader. Simulations were performed in order to optimize the design for the capacitor and channel, and to verify the change of S11.The micro-fluidic device was fabricated using an Su-8 micro-machining process. Measurements were performed using a VNA and a probe measurement station in order to verify the change in S11 with respect to the level of water in the micro-fluidic channel. The S11 measurements yield an $8 \mathrm{~dB}$ range which is in agreement with the theoretical calculations that are explained in the paper. For the radar echo measurements, a $4 \mathrm{dBm}$ range is obtained. This corresponds to a $10^{\circ} \mathrm{K}$ measurable temperature range. This design allows wireless temperature sensing at a distance, thus making it an effective solution for distant temperature monitoring applications.
\end{abstract}

\section{INTRODUCTION}

For various applications, such as product storage (nuclear waste, food, ect...) [1, 2], requiring small sized sensing devices, continuous and stable measurement and energetic autonomy, wireless passive sensors appear as a key technological solution [3]. Acoustic wave sensors have been one solution to overcome this need. However, the SAW sensor presents a limited reading distance.

In the past few years, a new generation of wireless passive sensors has emerged based on electromagnetic transduction and radar interrogation $[4,5]$. These type of sensors present many potential advantages. Adding to its battery less, low cost and long sensing range characteristics, the wireless passive monitoring technique presents direct modulation which allows a significant signal loss reduction.

\author{
M.M. Tentsiris \\ School of ECE, Georgia Tech, Atlanta, GA 30332-250, \\ U.S.A.
}

In this paper, we developed miniaturized, battery-less temperature sensor that can be interrogated at a distance by a radar. The temperature sensitive component will be based on micro-fluidic technology where liquid displacement within a micro-channel will be correlated to a temperature variation. This mechanical variation leads valuable changes of capacitor permittivity and thus variation of the component electromagnetic parameters. In that way the sensor uses a variable radar echo to wirelessly measure temperature at the Ka-frequency band. This integration of micro-fluidic and electromagnetic technology allows for component miniaturization and high sensitivity.

The design steps of the temperature micro-sensor will be presented in this paper followed by fabrication flow process and finally simulation and measurement results.

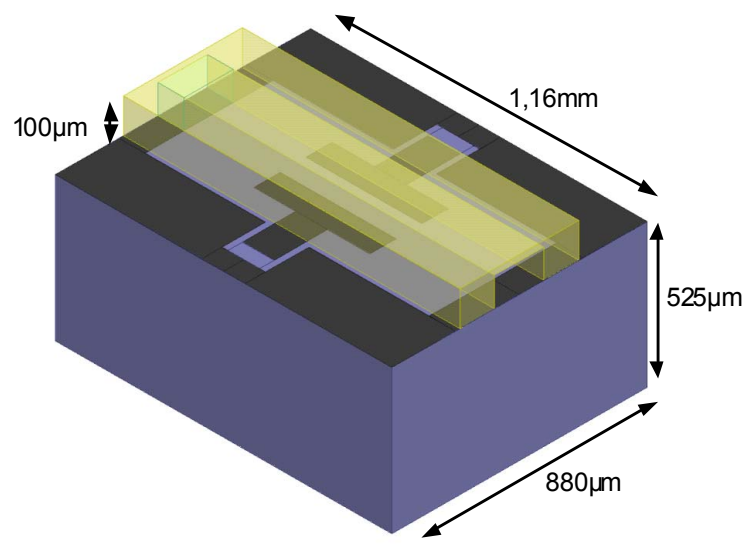

Fig. 1: Micro-fluidic Temperature micro-sensor.

\section{DEVICE DESCRIPTION}

The sensor structure in Fig. 1 is designed and optimized using Ansoft-HFSS. It consists of a micro-fluidic variable capacitor for wireless temperature monitoring in the millimeter-wave frequency band. The model consists of a coplanar gap capacitor with a water-filled micro-channel in between and above the plates. The liquid progressively fills the channel with the temperature-dependent volume expansion 
of water as seen in Fig 3. This rising level of high permittivity liquid within the capacitor modifies the scattering parameter S11. This sensor uses a variable radar echo to measure temperature at the frequency of $29,8 \mathrm{Ghz}$. This is done by connecting one access port of the sensor to a horn antenna with a 1 meter length $50 \Omega$ coaxial line while the other access port is loaded by $50 \Omega$ charge. the complete sensor system is interrogated by an FMCW radar.

The sensor structure is fabricated on $525 \mu \mathrm{m}$ thick glass substrate using a $100 \mathrm{~nm}$ thick gold layer and Su8 negative resin. The coplanar line was designed to be $50 \Omega$ with a $110 \mu \mathrm{m}$ wide center conductor and a $20 \mu \mathrm{m}$ wide spacing between the center and the ground planes. The dimensions of the planar capacitor plate $(90 \mu \mathrm{m}$ width, $400 \mu \mathrm{m}$ length and $90 \mu \mathrm{m}$ distance between plates) are optimized to increase the electric field between the capacitor plates. An Su-8 micro-channel filled with water $(170 \mu \mathrm{m} \times 100 \mu \mathrm{m})$ is then placed over the coplanar capacitor along the plates. With theses dimensions, a good sensitivity against water level position is obtained as well as a large temperature sensing range without compromising the capacitor performance. A large circular reservoir connected to the micro-channel is needed to provide the maximum liquid displacement, just as is done in thermometers. We use $10^{\circ} \mathrm{C}$ of temperature range (between $25^{\circ} \mathrm{C}$ and $35^{\circ} \mathrm{C}$ ) where the temperature dependant volume expansion of water is quite linear and evaporation is avoided. The necessary tank volume is calculated using the temperature-dependent volumetric expansion equation for a known spatial expansion coefficient of water and known dimensions of the micro-channel cross section:

$$
\frac{\Delta V}{V}=\alpha \Delta T
$$

Where $\mathrm{V}$ is the total volume, $\alpha$ is the volumetric thermal expansion coefficient, $\Delta \mathrm{V}$ is the fractional change in volume of liquid and $\Delta \mathrm{T}$ is the change in temperature.

\section{FABRICATION}

The sensor structures were fabricated in the clean-room of Laboratoire d'Analyse et d'Architecture des systems. Fig. 2 outlines the employed fabrication process. We review the main steps of the fabrication process.

The details and advantages of these fabrication techniques are elaborated on in detail by the authors in [4]. First, using a $525 \mu \mathrm{m}$ thick glass wafer, a $\mathrm{Ti} / \mathrm{Cu}(0.1 \mu \mathrm{m} / 0.1 \mu \mathrm{m})$ seed layer was sputtered on the both side. A lift-off method is used to pattern the planar capacitor structure with ground plane on the back side.

To improve the adhesion of the Su- 8 resin on glass, an adhesion promoter was spin coated on the wafer. On $100 \mu \mathrm{m}$ Su-8 negative resin, channels and tanks were then micromachined using photolithography.

Afterwards, using the Su-8/Su-8 lamination technique [6], a $50 \mu \mathrm{m}$ thick photosensitive Su- 8 dry film was placed on the top of the structure to close the structure and realize the fluidic access.

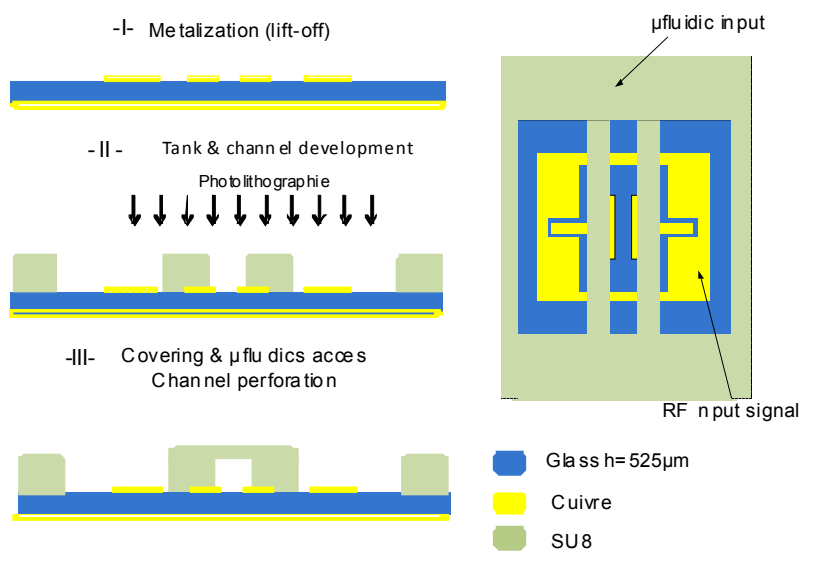

Fig. 2: Fabrication Process flow.

The next step was to fill the channel with water. First, the channel was sealed from one side with a square piece of glass and double-sided adhesive. Next, the structure was placed in a container filled with water and placed under vacuum so the

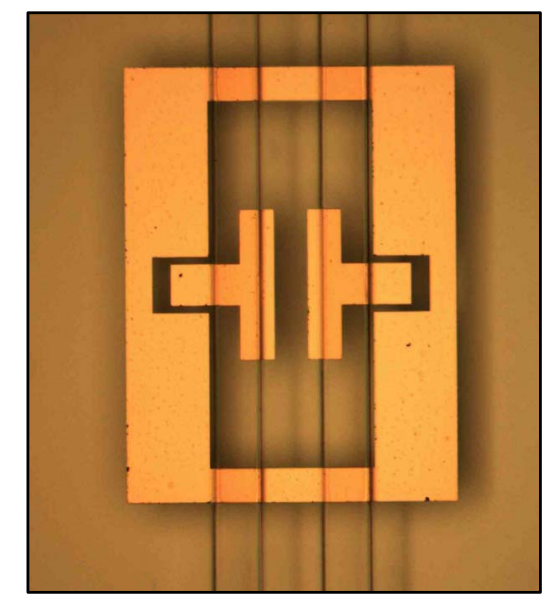

(a)
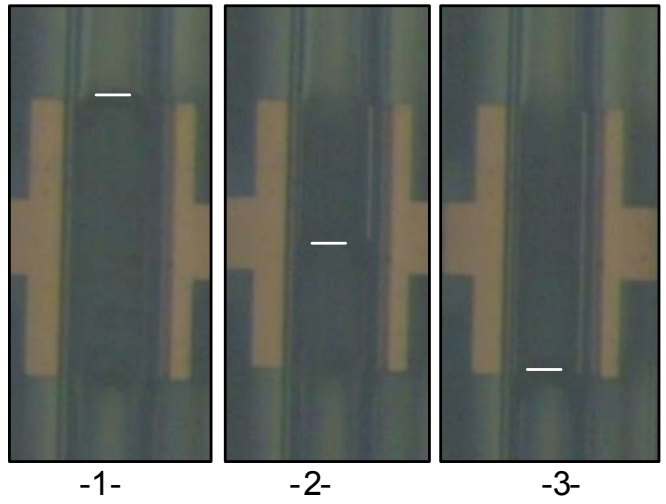

(b)

Fig. 3: (a) realized structure of the sensor (b) microchannel between the plates filled at b-1-at $0 \%$ b-2- at $50 \%$ and $b-3-$ at $100 \%$ with water. 
fluid was forced into the Su8 micro-structure using pressure.

Fig. 3 illustrates the realized micro-fluidic temperature sensor on (a) and three positions of water level inside the channel since the measurement on (b).

\section{MEASURED RESULTS}

Measurement of the scattering parameters were performed with a $70 \mathrm{Ghz}$ Anritsu Vector Network Analyzer connected to the probes in the borders of the sensor at a frequency of less than 40Ghz. In Fig. 4 the special RF/Temperature measurement bench is shown. It is composed of a classical $\mathrm{RF}$ measurement bench with a heating chuck.

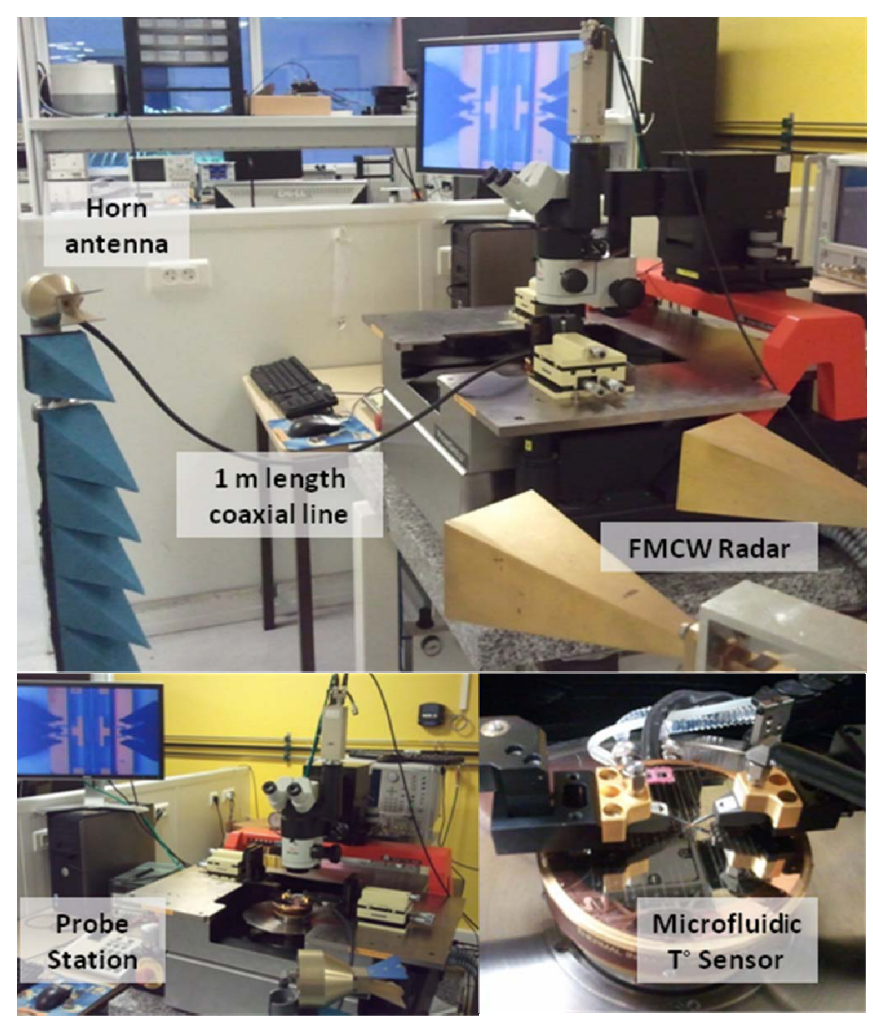

Fig. 4: On wafer RF/Temperature Measurement bench.

The radar echo level measurement of the device was accomplished using a 1 meter long coaxial probe connecting the standard $10 \mathrm{dBi}$ gain horn antenna to the sensor. To calibrate out resonances from the cable a one-port calibration was performed using short, open and load standards. The horn was then attached to this cable. Afterwards, on-wafer radar measurements were performed at $29,8 \mathrm{GHz}$ where the sensor was terminated by $50 \Omega$ [7]. The system is illuminated by an FMCW radar as shown in Fig. 5. The variation of the radar echo level is correlated to the temperature change.
In an FMCW radar, the waveform generated is continuous with time and is typically transmitted with $100 \%$ duty cycle to illuminate the area of observation. In that way, this interrogation technique allows a real time remote sensing at a high measurement range for applications where the temperature changes quickly.

Measurement results were obtained with respect to the liquid position in percentage of filling rate. For each point of measurement the liquid was stable in a precise position with $\pm 3 \mu \mathrm{m}$ of accuracy.

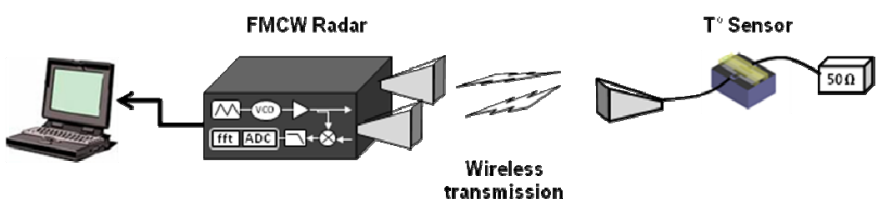

Fig. 5: Principle of the FMCW Radar EM sensor interrogation technique.

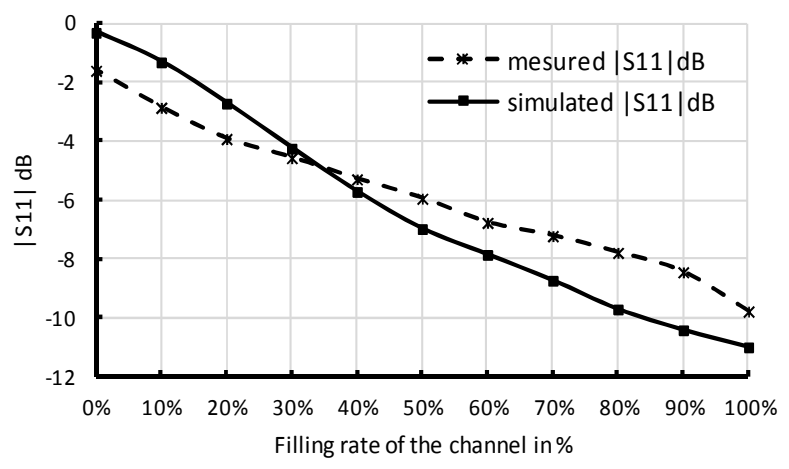

Fig. 6: Measured and Simulated reflection coefficient.

Using the VNA, the S parameter variations correspond to an $0.8 \mathrm{~dB} / \mathrm{deg}$ of sensitivity when associating the filling rate with the temperature expansion of water at a frequency of 29,8 Ghz.

Results obtained from the measurements are displayed in Fig. 6 and are in good agreement with simulation results. A slight constant offset is observed as a difference between simulation and measurement results. It is assumed as consequence of micro-fabrication effects and the environment disturbances which add parasitic capacitance. The measured is about $8 \mathrm{~dB}$ of variation for $10^{\circ} \mathrm{C}$ of temperature range.

In the other case, $4 \mathrm{dBm}$ radar echo level range is observed in the millimeter wave radar measurements. This difference in the radar echo level observed is sufficient to make a correlation with temperature change. The change in the applied physical value is converted into a linear decrease in radar echo level as shown in Fig. 7. The measured sensitivity is about $0,4 \mathrm{dBm} / \mathrm{deg}$. 


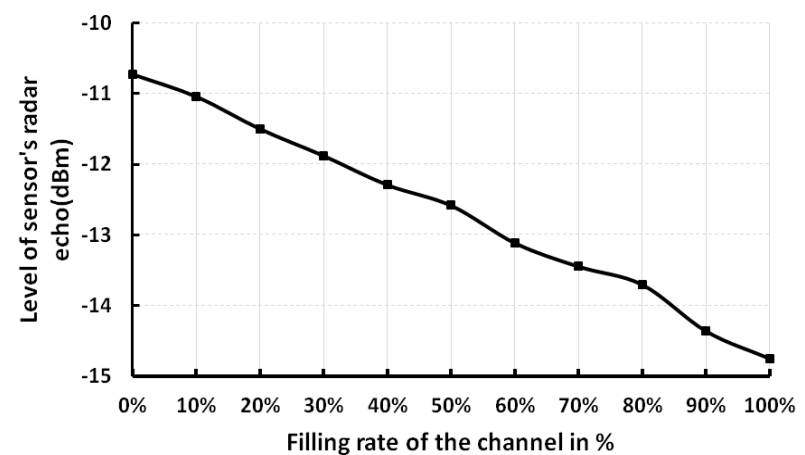

Fig. 7: Measured radar echo level of $\mathrm{T}^{\circ}$ sensor versus filling rate of the micro-channel.

\section{CONCLUSION}

A new micro-fluidic planar capacitor for temperature monitoring has been presented. The measurement principal, based on radar passive detection, has been demonstrated and the sensor performance of the fabricated fluidic microstructure has been measured. When tested using VNA and probes, $a 0.8 \mathrm{~dB} / \mathrm{deg}$ of sensitivity was obtained. Finally, the temperature sensor was integrated with a horn antenna via a 1 meter length coaxial delay line and was interrogated using the radar interrogation technique. Measured results obtained show a linear sensitivity of $0.4 \mathrm{dBm} / \mathrm{deg}$. This sensor demonstrates a reliable and cost effective solution to the challenges of realtime wireless temperature monitoring.

\section{ACKNOWLEDGMENT}

The authors would like to acknowledge the support of the Laboratoire d'Analyse et d'Architecture des Systèmes (LAASCNRS).

\section{REFERENCES}

[1] Emilio Sardini and Mauro Serpelloni, "Passive and SelfPowered Autonomous Sensors for Remote Measurements", Sensors 2009, 9, 943-960.

[2] Hande Alemdar, Cem Ersoy," Wireless sensor networks for healthcare: A survey", Computer Networks, Volume 54 (2010), pp 2688-2710.

[3] Luis Ruiz-Garcia, Loredana Lunadei, Pilar Barreiro and Jose Ignacio Robla, "A Review of Wireless Sensor Technologies and Applications in agriculture and Food Industry: State of the Art and Current Trends", sensors 2009, 9, 4728-4750.

[4] A. Traille, S. Bouaziz, S. Pinon, P. Pons, H. Aubert, A. Boukabache, et M. Tentzeris, "A wireless passive RCSbased temperature sensor using liquid metal and microfluidics technologies "), in Microwave Conference (EuMC), 2011 41st European, 2011, p. 45 -48.

[5] M. M. Jatlaoui, F. Chebila, T. Idda, P. Pons, et H. Aubert, "Phenomenological theory and experimental characterizations of passive wireless EM pressure microsensor prototype ", in 2010 IEEE Sensors, 2010, p. 643 646.

[6] Abgrall P, Lattes C, Conedera V, Dollat X, Colin S and Gue A M, "A novel fabrication method of flexible and monolithic 3D microfluidic structures using lamination of SU-8 films".

[7] M. M. Jatlaoui, F. Chebila, S. Bouaziz, P. Pons, et H. Aubert, « Original identification technique of passive EM sensors using loaded transmission delay lines $"$, in Microwave Conference (EuMC), 2010 European, 2010, p. $1106-1109$. 
